\section{Measuring pain self-efficacy and health related quality of life among hemodialysis patients in Greece: a cross-sectional study}

\section{Paraskevi Theofilou, ${ }^{1}$ Adamantia Aroni, ${ }^{2}$ Maria Tsironi, 3 Sophia Zyga ${ }^{3}$}

1Sotiria Hospital for Thoracic Diseases, Athens; ${ }^{2}$ General Hospital of Molaon, Sparta; ${ }^{3}$ Nursing Department, University of Peloponnese, Sparta, Greece

\begin{abstract}
Patients suffering from end-stage kidney disease often complain about pain. It is also known that the presence of chronic pain greatly impacts upon patients' quality of life (QOL) and can play a crucial role in the co-morbidity of mental health symptoms such as depression and anxiety. The main aim of this study protocol is the investigation of pain self-efficacy, QOL as well as their relation in patients undergoing hemodialysis treatment. The final sample size will be around 70-80 patients. Each subject's QOL and pain self-efficacy will be measured using the following instruments: i) the Missoula- ITAS Quality of Life Index-15 and ii) the Pain Self-Efficacy Questionnaire. QOL is expected to be related to pain self-efficacy scores. This probable association will be indicated performing regression as well as correlation analysis after controlling for gender, age, education and marital status.
\end{abstract}

\section{Background}

Chronic kidney disease (CKD) is a worldwide chronic disease associated with poor patient quality of life and mental health outcomes as well as high cost. ${ }^{1-4}$ As people with CKD age and present different comorbidities, they are also very likely to experience pain.5,6

Pain is defined as an unpleasant sensory and emotional experience associated with actual or potential tissue damage. ${ }^{7}$ Pain is a frequent complaint of CKD patients undergoing hemodialysis (HD), $8-10$ though it is not a common focus of research regarding this population. Specifically, information regarding its origins, frequency, and management is relatively scarce.

Most published data come indirectly from studies focusing on health-related quality of life.8,10 The reported frequency of pain varies widely in these patients. Murtagh et al., 11 in a review of symptoms in CKD, reported a weighted mean pain prevalence of $47 \%$, with a range of 8 to $82 \% .10$ Pain can arise in CKD from multiple causes, including surgery, and comorbidities such as osteoarthritis, ischemic limb disease and peripheral neuropathy. ${ }^{9}$ It is known that the presence of chronic pain greatly impacts upon quality of life (QOL) and can play a major role in the co-morbidity of anxiety and depression. 12 The aim of the present study is to investigate pain self-efficacy, QOL as well as their relation in patients undergoing $\mathrm{HD}$ treatment. Because of the fact that the pain selfefficacy questionnaire, which will be used in the context of this study, has not been evaluated before in Greece, we aim to investigate its psychometric properties as well.

\section{Methods}

A cohort of around 70-80 patients undergoing HD treatment will be recruited from hospitals located within the broader area of Peloponnese. The inclusion criteria are: i) $>18$ years of age; ii) ability of communication in Greek; iii) diagnosed with CKD; iv) HD treatment at least for a year; v) satisfying level of cooperation and perceived ability; vi) no history of primary psychiatric disease that may interfere with conduct of study; vii) clinically stable with no evidence of chronic or acute infections, inflammatory disorders, malignancy. Participants will be Greek adults having signed a consent form for participation. All subjects will be informed of their rights to refuse or discontinue participation in the study according to the ethical standards of the Helsinki Declaration. Ethical permission for the study will be obtained from the scientific committees of the participating hospitals.

The psychometric tools included in the study are presented below.

\section{Instruments}

Byock and Merriman created the MissoulaVITAS Quality of Life Index (MVQOLI). ${ }^{13}$ The MVQOLI is an assessment instrument that gathers patient-reported information about QOL during advanced illness. Maintaining optimal QOL is a core goal of palliative and hospice care, and information gathered via the MVQOLI assists health care professionals in identifying and addressing patient concerns that affect QOL. The MVQOLI has been used in many different healthcare settings including hospice, hospital, home health, long-term care (including assisted living), outpatient palliative care, disease management and pre-hospice programs. 13 The framework of the
Correspondence: Paraskevi Theofilou,

Sotiria Hospital for Thoracic Diseases, Athens, Greece.

Tel. $+30.697 .744 .1502,+30.210 .622 .1435$

Fax: +30.210 .622 .1435 .

E-mail: theofi@otenet.gr, paraskevi.theofilou@ gmail.com

Key words: pain self-efficacy, hemodialysis, quality of life, chronic kidney disease.

Contributions: the authors contributed equally.

Conflict of interests: the authors declare no potential conflict of interests.

Received for publication: 24 March 2013.

Revision received: 16 May 2013.

Accepted for publication: 20 May 2013.

This work is licensed under a Creative Commons Attribution NonCommercial 3.0 License (CC BYNC 3.0).

CC Copyright P. Theofilou et al., 2013

Licensee PAGEPress, Italy

Health Psychology Research 2013; 1:e30

doi:10.4081/hpr.2013.e30

MVQOLI is based on Ira Byock's work regarding growth and development at the end of life and the concepts of landmarks and tasks of life closure. ${ }^{13}$ The MVQOLI asks patients about 5 dimensions or domains of QOL: symptoms, function, interpersonal, well-being and transcendence. The instrument is specifically designed to assess the patients personal experience in each of these dimensions, hence the MVQOLI items are constructed with highly subjective language and no scores appear on the version of the tool seen by patients. The tool seeks to describe the qualitative and subjective experience of QOL in a way that can be quickly interpreted by professional caregivers. The scale has been translated and validated into Greek.14,15

The Pain Self-Efficacy Questionnaire (PSEQ) is a 10-item questionnaire developed to assess the confidence people with ongoing pain have in performing activities while in pain. It consists of two domains, physical and psychological. The PSEQ is applicable to all persisting pain presentations. It covers a range of functions, including household chores, socializing, work, as well as coping with pain without medication. 16

Demographic and clinical characteristics of all patients will be collected as baseline information at the beginning of the study.

\section{Data analysis}

Kolmogorov-Smirnov tests will be performed 
in order to check whether the values of the sample fell within a normal distribution. The analyses that will be used in the present study aim to investigate the relation between QOL and pain-self efficacy in HD patients. Thus, correlation analysis will be performed using Pearson's rho. Hierarchical regression analyses will be also used to assess the above association in the total sample. Statistical analyses will be performed with the use of independent samples $t$ test and one-way ANOVA in order to investigate potential effects of socio-demographic factors on QOL and pain self-efficacy. Finally, independent samples $\mathrm{t}$ test analysis will be used in order to examine differences between patients who recently commenced treatment $(<4$ years) and those on long term treatment ( $>4$ years). A P value of 0.05 or less will be considered to indicate statistical significance. All analyses will be performed with the Statistical Package for the Social Sciences (SPSS 13.0 for Windows). Two health psychologists will select the data using the relevant psychometric tools in the context of an interview at clinic in order to avoid missing data.

\section{Discussion}

This study will ascertain the association between QOL and pain self-efficacy in HD patients. The findings of the present study can be used in the development of health care services and in-patient management. The role of QOL and pain self-efficacy in particular may play an important role in the course of illness and treatment outcomes and could therefore be identified as a new area for psychological intervention in people with CKD.17,18

\section{References}

1. Theofilou P, Panagiotaki H. Comparative study of bicarbonate dialysis and haemodiafiltration in end-stage renal disease. In: Theofilou P, ed. Outcomes assessment in end-stage kidney disease - measurements and applications in clinical practice. Sharjah: Bentham Science Publishers. [In press]

2. Theofilou P. Association of insomnia with kidney disease quality of life reported by patients on maintenance dialysis. Psychol Health Med 2013;18:70-8.

3. Theofilou P. Self-reported functional status: an important predictor of mental health outcomes among chronic dialysis patients. Eur J Psychol Assess 2012. [In press].

4. Theofilou P. Quality of life and mental health in hemodialysis and peritoneal dialysis patients: the role of health beliefs. Int Urol Nephrol 2012;44:245-53.

5. Davison SN. Chronic kidney disease: psychosocial impact on chronic pain. Geriatrics 2007;62:17-23.

6. Parrino I, Casuccio A. Incidence of chronic pain in patients with end-stage renal disease on dialysis. J Pain Symptom Manag 2005;30:302-4.

7. International Association for the Study of Pain. Classification of chronic pain. 2nd Ed. Seattle: IASP Press; 1994.

8. Davison SN. Pain in hemodialysis patients: prevalence, cause, severity and management. Am J Kidney Dis 2003;42: 1239-47.

9. Mercadante S, Ferrantelli A, Tortorici C, et al. Incidence of chronic pain in patients with end-stage renal disease on dialysis. $\mathrm{J}$ Pain Symptom Manag 2005;30:302-4.
10. Golan E, Haggiag I, Os P, Bernheim J. Calcium, parathyroid hormone, and vitamin D: major determinants of chronic pain in hemodialysis patients. Clin J Am Soc Nephrol 2009;4:1374-80.

11. Murtagh FE, Addington-Hall J, Higginson IJ. The prevalence of symptoms in end stage renal disease: a systematic review. Adv Chronic Kidney Dis 2007;14:82-99.

12. Gureje 0, Korff M, Simon G, Gater, R. Persistent pain and well-being: a World Health Organization study in primary care. JAMA 1998;280:147-51.

13. Schwartz CE, Merriman M, Reed G, et al. Evaluation of the Missoula-VITAS Quality of Life Index-Revised: research tool or clinical tool? J Palliative Med 2005;8:121-35.

14. Theofilou P, Kapsalis F, Panagiotaki H. Greek version of MVQOLI-15: translation and cultural adaptation. Int $\mathrm{J}$ Caring Sci 2012;5:289-94.

15. Theofilou P, Aroni A, Ralli M, et al. Measuring health-related quality of life in haemodialysis patients: psychometric properties of the Missoula-VITAS Quality of Life Index (MVQOLI-15) in Greece. Health Psychology Research 2013. [In press].

16. Nicholas MK. Self-efficacy and chronic pain. Paper presented at the annual conference of the British Psychological Society. St. Andrews, 1989.

17. Rebollo P, Ortega F, Baltar JM, et al. Health-related quality of life (HRQOL) in end stage renal disease (ESRD) patients over 65 years. Geriatr Nephrol Urol 1998:8:85-94.

18. Ellinikou M, Zissi A. Quality of life and chronic illnesses. Prognostic variables for the psychosocial rehabilitation of the patients with chronic illness. Medicine 2002;82:124-31. [Article in Greek]. 